\title{
Relationship between Star-Wave Test and Visual/Motor Maturity in Young Children
}

\author{
Shogo Komatsu \\ Kanagawa Prefectural General Education Center, Kanagawa, Japan \\ Email: look.what.happened.7700@gmail.com
}

How to cite this paper: Komatsu, S. (2019) Relationship between Star-Wave Test and Visual/Motor Maturity in Young Children. Open Journal of Nursing, 9, 173-182. https://doi.org/10.4236/ojn.2019.92016

Received: December 29, 2018

Accepted: February 19, 2019

Published: February 22, 2019

Copyright $\odot 2019$ by author(s) and Scientific Research Publishing Inc. This work is licensed under the Creative Commons Attribution International License (CC BY 4.0).

http://creativecommons.org/licenses/by/4.0/

\section{c) (i) Open Access}

\begin{abstract}
The developmental maturity (M) scale using the Star-Wave Test that was independently developed by Yalon and Zion indicates poor learning abilities if the M-scale score is low, which may be caused by developmental delays, such as intellectual disability and learning disorder or organic mental disorder. Furthermore, the distress (D) scale is thought to be influenced by mild neurological disorders, hand coordination problems, and environmental factors. In this study, we conducted a Star-Wave Test on 44 nursery school children in Japan and examined the $\mathrm{M}$ scale, the $\mathrm{D}$ scale, as well as the level of stress induced by coordination problems and the environment. The t-test was conducted on the $\mathrm{M}$ scale and $\mathrm{D}$ scale of the group without coordination problems and that with coordination problems, and we found a significant difference at $1 \%$ level. From this result, we can confirm the relationship of the $\mathrm{M}$ scale and the D scale with Visual and motor maturity.
\end{abstract}

\section{Keywords}

Star-Wave Test, Projection Drawing Method, Infancy,

Developmental Maturity Scale, Distress Scale

\section{Introduction}

Star-wave test is to draw "ocean waves and starry sky" with pencils on the paper with a printed $10.5 \times 15.3 \mathrm{~cm}$ rectangle frame. [1] mainly proposed handwriting analysis for the screenings as "developmental functional test" for preschool children. After a while, he found out that personality characteristic can be grasped by introducing Jungian psychology, and began to use regardless of the ages.

The drawing analysis consists of 3 handwritten elements, 1) wave movement, 2) shape of star, 3) spatial arrangement. Instead, Lallemant proposed following 5 
steps for analysis:

1) Drawing classification: a) only points b) painterly c) emotional d) formal e) symbolic, total 5 types.

2) Structure of space: a) harmony b) apposition c) regularity d) disharmony, total 4 types.

3) The symbolic expression of space: 7 kinds of analysis for the vertical direction, 4 kinds of analysis for horizontal analysis. The vertical direction is classified into a) harmony of sky and sea, b) sky's predominance, c) sea's predominance, d) sky and sea on the horizon, e) the gap between sky and sea, f) emphasized space between sky and sea, g) mixed; horizontal analysis is classified into i) no emphasis, ii) emphasis on the left iii) emphasis on the middle iv) emphasis on the right.

4) Symbol of things: Analyze symbolic meanings of stars, waves, rocks and cliffs, islands, boats, moon, coast, lighthouse and so on.

5) Handwriting: There are 6 ways of drawing lines: Single line, shaking line, stable line, unstable line, continuous line, and shredding line. There are 8 types to stroke: General lines (delicate lines, sharp lines, soft lines, and solid lines), lines with signs of obstacles (narrow lines, hard lines, fragile lines, and cluttered lines).

Additionally, we analyze whether there is a black trace of stuck handwriting and trace of handwriting falling apart as a sign of disability. As a research of star-wave test in Japan, there is "star-wave test trial in Japan (as a developmental functional test for school children)" by [2].

[3] released "star-wave test trial in Japan" in the bulletin 24 of basic science, Nippon medical school in 1998; also "star-wave test as a development functional test: Drawing by Kindergarten Children" as a document in 1999 [4]. [5] published the book "star-wave test introduction" in 2000.

[6] published the book "projection drawing method test battery star-wave test/Walteg Drawing Test/Baum Test” advocated by Avé-Lallemant in 2012. Thus, the practice and research of SWT has undergone various developments during the past quarter century. As one of them, [7] from Israel published "The Star-Wave Test across the Life Span-Advances in Theory", Research and Practice in 2006. Together with Zion (Ben-Zion), Yalon conducted SWT on preschool children and created maturity (M) scale and distress (D) scale. These scales were not created to conclude and label disabled children but created to find children who need individual support sooner and provide the support. This $\mathrm{M}$ scale can be used as an evaluation standard of general development skills necessary for school education. And the low score reflects underdeveloped learning ability which could mean intellectual impairment, learning disability, as well as the developmental delay or the existence of organ problems. Also, the quality of waves is said to be able to predict the difficulty of academic performance and sociality, especially writing disturbance. As for D scale, the high score means that the child is suffering from stress at least for a certain period of time. The reasons 
are as follows: 1) physical: mild neuropathic disorder, low muscle tension, clumsy pencil holding etc. 2) environmental: neglect, divorce, bereavement, etc. 3) psychological: a decline in self-esteem, anxiety, perfectionism, etc.

Yalon and Zion retested in a few years to conduct an empirical study to predict the risk of atypical development (developmental disorder), suggesting its possibility.

The Star-Wave Test is a simple paper-based test that can be taught easily, which enables its group-based implementation for preschool children and indicates its effectiveness as a screening test. Moreover, the use of the $\mathrm{M}$ scale and the D scale enables quantitative evaluation of the level of developmental functions of preschool children.

However, there have been almost no studies conducted on what kind of abilities of young children that can be related to the $\mathrm{M}$ scale and the $\mathrm{D}$ scale proposed by Yalon and Zion as SWT's developmental functions.

\section{Objective}

The $M$ scale is thought to be related to general development skills, and the $D$ scale is thought to be related to mild neurological disorders, low muscle tension, and a clumsy way to hold a pencil. The D scale is also believed to be influenced by environmental stresses and each individual's abilities. In this study, we conducted a Star-Wave Test for children in a nursery school and compared the relationship between the $\mathrm{M}$ scale and the $\mathrm{D}$ scale, which were proposed by Yalon and Zion, with exercise coordination problems and home environmental factors by using the modified version of Avé-Lallemant's personal check tables. We also evaluated the features of the drawings by a child with cerebral palsy, whose motor functions are impaired.

\section{Method}

\subsection{Survey Subjects and Schedule}

SWT was conducted on a total of 44 subjects, consisting of 23 five-year-old children and 21 six-year-old children, who attended A nursery school in February 2018. The nursery teachers of A nursery school, who conducted the SWT to the 44 subjects, were handed out excerpts of Avé-Lallemant's personal check tables and evaluated the subjects. The contents of the evaluation include whether or not clumsiness was observed in daily tasks, such as handling small items, hand motoric skills, etc., and whether there were stresses from the home environment.

\subsection{Survey Tools}

For the test implementation, the children were given an SWT paper (the size of the inside frame is $15.3 \times 10.3 \mathrm{~cm}$ with a $1-\mathrm{mm}$ frame), an $\mathrm{HB}$ pencil, an eraser, and a pencil sharpener. Personal check tables were also prepared for the nursery teachers. 


\subsection{Procedures}

The SWT was conducted in February 2018 collectively at A nursery school. The testers were two clinical psychologists and two assistants. During the test implementation, the testers started with asking the subjects with the following questions: "Do you know the stars in the sky? Do you know the waves on the ocean?", followed by the following instruction: "Please draw the stars and the ocean waves". Beforehand, the personal check tables (number, date of birth, ages in years and months, special mentions, and others), and numbered each subject without exposing any names. As an ethical consideration, only the nursery school can identify the individual based on the identification number, and the investigators were not informed. The data is stored in a lockable locker for five years.

\subsection{Analysis Method}

Drawing judgment was conducted on 44 subjects by 2 clinical psychologists who did the research on drawing and regularly used drawing test at the clinical site. After they calculated $\mathrm{M}$ scale and D scale of individual drawing, they consulted and confirmed the scores at the meeting. Scoring methods for $\mathrm{M}$ scale and $\mathrm{D}$ scale are as follows.

"Scoring method for M scale"

Calculate from 5 items (Table 1). Highest score total 10 points, average score 6 points, and less than 5 points are a low score.

1) Comprehension of task: Whether star and wave are drawn or not. If yes, the score is 2 points, 1 point if partially drawn, if not 0 points (same applies as below).

2) Shape of a star: 2 points if drawn, 1 point for round star or circled star.

3) Wave movement: 2 points if drawn. 1 point if linear, awkward, and arch type

4) Spatial arrangement: 2 points for stars on the upper part, waves of the sea on the lower part, 1 point if partially drawn.

5) Acknowledgment of the frame, the highest score 2 points. The qualitative level is the highest score 2 points.

a) Acknowledgment of frame: 1 point if drawn within the frame and drawn out of the frame only one time. 0 point if drawn outside of the frame more than 2 times.

b) Qualitative level: 1 point for preliminary characteristics (sustainability, recognition, effort, self-confidence), 0 points for lack of preliminary characteristics, disturbance factors (laziness, abandonment, negligence, inactivity, dreamy).

6) Comprehension of task: Whether star and wave are drawn or not. If yes, the score is 2 points, 1 point if partially drawn, if not 0 points (same applies as below).

7) Shape of a star: 2 points if drawn, 1 point for round star or circled star.

The word "data" is plural, not singular. 
Table 1. The maturity scale.

\section{The Maturity Scale}

\section{Task Understanding}

2 -stars placed over the wave with different, recognisable forms

2-relevant additions (fish, boat, moon, etc.)

1 -stars only

1 -waves only

1 -just one star, huge and centrally placed

1 -irrelevent additions (flower, butterfly, sun, etc.)

0 -another drawing

0 -an empty sheet

\section{Form of Stars}

2-at least one well-formed angular star

1-a planned angular form, with malformations due to impaired movement

1 -round stars, good circles

0 -form disturbances

0 -scribbles, no forms

0-lack of stars

\section{Movement of Waves}

2-at last one rhythmically wave

1-linear or angular (zigzag) strokes

1 -arcades

1-other static forms

0 -movement disturbances (sometimes only in the waves)

0 -blacking of wave-area

0 -lack of waves

\section{Spatial Arrangement}

2-stars placed over waves with good macrostructure

2 -stars over waves with stars placed in a row (typical for children at age five)

1-upper part with stars, lower part empty

1-disturbed macrostructure

0 -spatial problems: waves over stars, or side by side

0 -stars all over the sheet

0 -waves all over the sheet

0 -stars and waves on one side of the frame only

\section{Frame Recognition}

1-drawing within the frame, or one deviation

0 -two deviations or more outside the frame

\section{Qualitative Level}

1-anxiliary qualities (persistence, ideas, diligence, self-asurance, or any trait that might help coping in school)

0 -lack of such auxiliay qualities, or even occurrence of disturbing factors (indolence, giving up, slackness, dullnes, fantasm, etc.)

8) Wave movement: 2 points if drawn. 1 point if linear, awkward, and arch type.

9) Spatial arrangement: 2 points for stars on the upper part, waves of the sea on the lower part, 1 point if partially drawn.

10) Acknowledgment of the frame and qualitative level, 2 points in both.

a) Acknowledgment of frame: 1 point if drawn within the frame and drawn out of the frame only one time. 0 point if drawn outside of the frame more than 2 times. 
b) Qualitative level: 1 point for preliminary characteristics (sustainability, recognition, effort, self-confidence), 0 points for lack of preliminary characteristics, disturbance factors (laziness, abandonment, negligence, inactivity, dreamy).

"Scoring method for D scale"

Calculate from 5 items, subordinate items are 21 items (Table 2). Total 42 points, average 5 points, and more than 6 points are a high score.

1) Primary sign indicating weakness (loose movement, etc. 4 items).

2) Primary signature of uncontrollable impulses (3 items such as fragile handwriting).

3) Secondary sign of tension (7 items such as narrowing).

4) Secondary signature of over-guarantee control (high regularity, etc. 3 items).

5) The sign of environmental maladjustment (4 items such as discordant space) for above 21 items, 2 points if it is strong or high frequency, and 1 point if it is weak or up to 2 times.

\section{Results}

The average values of $\mathrm{M}$ scale and D scale of the 44 nursery school children subjects are shown in Table 3.

1) Examination on the relationship of the $M$ scale and D scale with "clumsiness"

By evaluation by nursery teachers, there were 9 infants who recognized "clumsiness". The t-test was done to determine whether there was a significant difference in the mean values of the M scale between the group in which "clumsiness" was observed ( 9 subjects) and the group in which "clumsiness" was not observed (35 subjects). As a result, a significant difference was observed at $1 \%$ level, in which the group with observed "clumsiness" had a significantly lower M-scale score $(\mathrm{t}(10)=3.864 \mathrm{p}<0.01)$

Furthermore, the t-test was also used to determine whether there was a significant difference in the mean values of the $\mathrm{D}$ scale between the group in which "clumsiness" was observed (9 subjects) and the group in which "clumsiness" was not observed (35 subjects). As a result, a significant difference was observed at $1 \%$ level, in which the group with observed "clumsiness" had a significantly lower $\mathrm{D}$-scale score $(\mathrm{t}(10)=4.061 \mathrm{p}<0.01)$. The results are shown in Table 4 .

2) Examination on the relationship of the $M$ scale and $D$ scale with "home environmental factors"

By evaluation by nursery teachers, there were 13 infants who recognized "stress in the home environment". The t-test was done to determine whether there was a significant difference in the mean values of the $M$ scale between the group in which "stress due to home environments" was present (13 subjects) and the group in which "stress due to home environments" was not present (31 subjects). As a result, no significant differences were observed $(\mathrm{t}(20)=0.300 \mathrm{p}<$ $0.01)$. 
Furthermore, the t-test was also used to determine whether there was a significant difference in the mean values of the $\mathrm{D}$ scale between the group in which "stress due to home environments" was present (13 subjects) and the group in which "stress due to home environments" was not present (31 subjects). As a result, no significant differences were observed $(t(16)=2.262 \mathrm{p}<0.01)$. The results are shown in Table 5 .

3) Case example

A child with cerebral palsy, no delay in intellectual ability, female, six years and nine months old.

M-scale score 2 points, $\mathrm{D}$-scale score 13 points.

The subject had difficulties in drawing lines. The shapes of stars and waves were not clear, and it was difficult to identify what the subject had drawn.

Table 2. The distress scale.

\section{The Distress Scale}

\section{SIGNS OF DISTRESS}

Primary Signs (Weakness)

1) Slack motion

2) Slack, non-elastic stroke

3) Fragile stroke

4) Stroke interruption (uncontrolled breaks)

Primary Signs (Uncontrolled Drives)

5) Spongy stroke

6) Crude stroke

7) Hyperkinesis

Secondary Signs (Tension)

8) Narrowness

9) Cover Strokes

10) Contracted movement

11) Tense, non-elastic stroke

12) Hard stroke

13) Corrections by retouching and easing

14) Fixational blackening

Secondary Signs (Overcompensating Control)

15) Very regular arrangement

16) Stylisation, from interpretation of the test

17) Hatching of the plane

Maladjustment to the Environment

18) Disrupted space, disharmonious, empty areas

19) Entanglements

20) Final blocking

21) Roughing of the pla

Table 3. The average scores for M scale and D scale of 44 nursery school children.

\begin{tabular}{lc}
\hline & nursery school children $(\mathrm{n}=44)$ \\
\hline M scale & 6.98 \\
D scale & 5.59 \\
\hline
\end{tabular}


Table 4. A t test of a group in which clumsiness is observed and a group in which clumsiness is not observed.

\begin{tabular}{|c|c|c|c|}
\hline & $\begin{array}{l}\text { a group in which clumsiness } \\
\text { is observed }(n=9)\end{array}$ & $\begin{array}{l}\text { a group in which clumsiness is } \\
\text { not observed }(n=35)\end{array}$ & \\
\hline & $\mathbf{M}(\mathrm{SD})$ & $M(S D)$ & $\mathrm{t}$ \\
\hline M scale & $4.22(2.54)$ & $7.69(1.75)$ & $3.86^{*}$ \\
\hline D scale & $8.44(2.51)$ & $4.86(1.70)$ & $4.06^{*}$ \\
\hline
\end{tabular}

${ }^{*} \mathrm{p}<0.01$.

Table 5. A t test of a group in which "stress due to home environments" was present and the group in which "stress due to home environments" was not present.

\begin{tabular}{cccc}
\hline & $\begin{array}{c}\text { a group in which "stress due a group in which "stress due to } \\
\text { to home environments" was } \\
\text { present }(\mathrm{n}=13)\end{array}$ & $\begin{array}{c}\text { home environments" was not } \\
\text { present }(\mathrm{n}=31)\end{array}$ & $\mathrm{t}$ \\
\hline $\mathrm{M}(\mathrm{SD})$ & $\mathrm{M}(\mathrm{SD})$ & 0.30 \\
M scale & $7.15(2.61)$ & $6.90(2.30)$ & 0.26 \\
\hline D scale & $5.77(3.24)$ & $5.52(1.95)$ & $\mathrm{t}$ \\
\hline
\end{tabular}

${ }^{*} \mathrm{p}<0.01$.

\section{Consideration}

1) Examination on the relationship of the $M$ scale and D scale with "clumsiness"

In the group where clumsiness was observed, the M-scale and D-scale scores were significantly lower and higher, respectively. A report on SWT on 721 German kindergarten children by [1] suggested that the test can be completed by children aged five years old, except in some cases with developmental delays or disabilities. In addition, when the subject's function matures and becomes fully compliant with the test, the test can also be used to detect any restrictions or problems based on the drawn picture, which is shown more clearly for children at the age of 6 and 7 .

[5] described the relationship between the inability to properly draw a star and the decline in visual-motoric ability in alcohol-dependent patients with impaired brain functions.

In addition, according to [8], in theBender Visual Motor Gestalt Test, which requires the ability to reproduce shapes accurately, it is thought that an immature or dysfunctional visual-motoric ability can be suspected if the Bender score is higher than the control group by $1 \mathrm{SD}$ or more. Also, based on a study on developmental functions reported by [2], the Bender Visual Motor Gestalt Test and the Star-Wave Test are considered to share a common point in producing expressions based on motoric functions, and the Star-Wave Test requires the internal generation of the "star" and "wave" concepts before the subjects can produce the expressions based on motoric functions.

Based on the data in this study and other studies, the M scale and the D scale have been shown to be related to brain functions, in particular, the visual-motoric 
functions that are required to draw appropriate stars and smooth waves.

2) Examination on the relationship of $M$ scale and D scale with "home environmental factors"

There were no significant differences of the $\mathrm{M}$-scale and the $\mathrm{D}$-scale scores in the group in which stresses due to home environmental factors were observed and that in which stresses due to home environmental factors were not observed. However, clumsiness is considered to be a variable that can be evaluated by daily observations of nursery school children. On the other hand, stress due to home environmental factors is an event that happens within the subject's family that it is difficult to observe by a childcare professional, making its evaluation difficult.

3) Case example

For the case example, although the subject had cerebral palsy with no delay in intellectual ability, the M-scale score was lower than average, and the D-scale score was higher than average. Therefore, we suggest that both the $\mathrm{M}$ scale and the D scale strongly reflect the influence of the motor functions in preschool children.

\section{Summary and Future Remarks}

We have confirmed in this study that the M scale and D scale, which were proposed by Yalon and Zion, are related to Visual and motor maturity. However, we could not confirm the relationship between the D scale and the level of stress from the environment. Therefore, there is a limit in measuring the level of stress from the environment of preschool children using this method of evaluation alone, implying that other methods need to be developed in future studies. In addition, although Yalon and Zion suggested the relationship between the M scale and learning disabilities, such a relationship has not been confirmed in this study it. Thus, in order to apply SWT as a method for early detection of learning disabilities in order to provide support for children with such disabilities, it is necessary to perform future verifications by using not only the $\mathrm{M}$ scale and the D scale but also other drawing features.

\section{Acknowledgements}

I would like to thank Ms. Kyoko Sugiura, Mr. Ryuta Kanemaru, Mr. Yasuaki Suzuki and Ms. Tomoko Aoki for their advice and guidance to conclude this thesis.

\section{Conflicts of Interest}

The author declares no conflicts of interest regarding the publication of this paper.

\section{References}

[1] Lallemant, A.U. (1984) The Star-Wave-Test. Studienkreis Ausdruckswissenschaft, Munchen. 
[2] Kyoko, S., Yasuaki, S., Hideto, M. and Kaoru, N. (1998) Star-Wave Test Trial in Japan-As a Developmental Functional Test for School Children. Research-Aid Paper of the Yasuda life Welfare Foundation, 102-103.

[3] Kyoko, S. and Hideto, M. (1998) Star-Wave Test Trial in Japan. The Bulletin 24 from Japan Medical University Basic Science, 27, 5-32.

[4] Kyoko, S. and Hideto, M. (1999) The Star Wave Test as a Developmental Functional Test: Drawing by Kindergarten Children. The Bulletin 27 from Japan Medical University Basic Science, 27, 19-43.

[5] Rhyner, B., Kyoko, S. and Yasuaki, S. (2000) Star-Wave Test Introduction. Kawashima Bookstore, Tokyo.

[6] Kyoko, S. and Ryuta, K. (2012) Projection Drawing Method Test Battery Star-Wave Drawing Test/Walteg Drawing Test/Baum Test. Kawashima Bookstore, Tokyo.

[7] Yalon, D. (2006) The Star-Wave Test across the Life Span-Advances in Theory, Research, and Practice. International Graphological Colloquium, Canada.

[8] Bender, L. (1938) A Visual-Motor Gestalt Test and Its Clinical Use. American Ortho Psychiatric Association Monograph Series Number 3. American Orthopsychiatric Association, NY. 